\title{
EXISTENCE OF THREE SOLUTIONS FOR A CLASS OF NAVIER QUASILINEAR ELLIPTIC SYSTEMS INVOLVING THE $\left(p_{1}, \ldots, p_{n}\right)$-BIHARMONIC
}

\author{
LiN Li
}

\begin{abstract}
In this paper, we establish the existence of at least three solutions to a Navier boundary problem involving the $\left(p_{1}, \ldots, p_{n}\right)$-biharmonic systems. We use a variational approach based on a three critical points theorem due to Ricceri [B. Ricceri, A three critical points theorem revisited, Nonlinear Anal. 70 (2009), 3084-3089].
\end{abstract}

\section{Introduction and main results}

In this work, we study the existence of at least three weak solutions for the nonlinear elliptic equation of $\left(p_{1}, \ldots, p_{n}\right)$-biharmonic type under Navier boundary conditions:

$$
\begin{cases}-\Delta\left(\left|\Delta u_{1}\right|^{p_{1}-2} \Delta u_{1}\right)=\lambda F_{u_{1}}\left(x, u_{1}, \ldots, u_{n}\right)+\mu G_{u_{1}}\left(x, u_{1}, \ldots, u_{n}\right) & \text { in } \Omega, \\ -\Delta\left(\left|\Delta u_{2}\right|^{p_{2}-2} \Delta u_{2}\right)=\lambda F_{u_{2}}\left(x, u_{1}, \ldots, u_{n}\right)+\mu G_{u_{2}}\left(x, u_{1}, \ldots, u_{n}\right) & \text { in } \Omega, \\ \cdots & \\ -\Delta\left(\left|\Delta u_{n}\right|^{p_{n}-2} \Delta u_{n}\right)=\lambda F_{u_{n}}\left(x, u_{1}, \ldots, u_{n}\right)+\mu G_{u_{n}}\left(x, u_{1}, \ldots, u_{n}\right) & \text { in } \Omega, \\ u_{i}=\Delta u_{i}=0 \text { for } 1 \leq i \leq n, & \text { on } \partial \Omega,\end{cases}
$$

where $\lambda, \mu \in[0,+\infty), \Omega \subset \mathbb{R}^{N}(N \geq 1)$ is a non-empty bounded open set with a sufficient smooth boundary $\partial \Omega, p_{i}>\max \left\{1, \frac{N}{2}\right\}$ for $1 \leq i \leq n$. $F$, $G: \Omega \times \mathbb{R}^{n} \mapsto \mathbb{R}$ are functions such that $F\left(\cdot, t_{1}, \ldots, t_{n}\right), G\left(\cdot, t_{1}, \ldots, t_{n}\right)$ are measurable in $\Omega$ for all $\left(t_{1}, \ldots, t_{n}\right) \in \mathbb{R}^{n}$ and $F(x, \cdot), G(x, \cdot)$ are continuously

Received March 12, 2011.

2010 Mathematics Subject Classification. 35J48, 35J60, 47J30, 58E05.

Key words and phrases. $\left(p_{1}, \ldots, p_{n}\right)$-biharmonic, Navier condition, multiple solutions, three critical points theorem.

Supported by the Fundamental Research Funds for the Central Universities (No. XDJK2013D007), Scientific Research Fund of SUSE (No. 2011KY03) and Scientific Research Fund of SiChuan Provincial Education Department (No. 12ZB081). 
differentiable in $\mathbb{R}^{n}$ for a.e. $x \in \Omega$. Moreover, $G$ satisfies the condition

$$
\sup _{\left|\left(t_{1}, \ldots, t_{n}\right)\right| \leq s} \sum_{i=1}^{n}\left|G_{t_{i}}\left(x, t_{1}, \ldots, t_{n}\right)\right| \leq h_{s}(x)
$$

for all $s>0$ and some $h_{s} \in L^{1}$ with $G(\cdot, 0, \ldots, 0) \in L^{1} . F_{i}$ denotes the partial derivative of $F$ with respect to $i, 1 \leq i \leq n$, so does $G_{i}$.

In recent years, the three critical points theorem of B. Ricceri has been widely used to solve differential equations (see $[2,4,6,8,10,13,14,15,21,23]$ and references therein). Using the three critical points theorem, some authors have considered the elliptic systems. In [12], Li and Tang get three solutions for a class of quasilinear systems involving the $(p, q)$-Laplacian with Dirichlet boundary condition. Afrouzi and Heidarkhani [1] unify and generalize Li and Tang's problem. In [8], El Manouni and Kbiri Alaoui consider $(p, q)$-Laplacian systems with Neumann conditions via Ricceri's three critical points theorem. $\mathrm{Li}$ and Tang [14] consider a $(p, q)$-biharmonic system under Navier boundary condition. In [10], Heidarkhani and Tian study a class of gradient systems depending on two parameters, they get three solutions using Ricceri's three critical point theorem. Later, Heidarkhani and Tian [11] using the same method study a class of gradient Kirchhoff-type systems depending on two parameters. Graef, Heidarkhani and Kong [9] get multiplicity results for multi-point boundary value problems.

There seems to be increasing interest in studying fourth-order boundary value problems, because the static form change of beam or the sport of rigid body can be described by a fourth-order equation, and specially a model to study traveling waves in suspension bridges can be furnished by the fourth-order equation of nonlinearity, so it is important to Physics. More general nonlinear fourth-order elliptic boundary value problems have been studied $[5,7,16,17]$. Particularity, Li and Tang [13] consider the $p$-harmonic equation with Navier boundary condition. Using the three critical points theorem of B. Ricceri, they get at least three solutions. Recently, Li and Tang [14] also use three critical points theorem to study a class of $(p, q)$-biharmonic systems. Here, as in [14], our main tool is Ricceri's three critical points theorem; see Theorem 2.1 in the next section. We also recall that, again applying Ricceri's three critical points theorem, elliptic systems have been studied in $[3,4,9,10,11]$. The aim of the present paper is to extend the main result of [14] to the general case.

In this paper, precisely we deal with the existence of an open interval $\Lambda \subseteq[0,+\infty)$ and a positive real number $\rho$ with the following property: for every $\lambda \in \Lambda$ and an arbitrary function $G: \Omega \times \mathbb{R}^{n} \rightarrow \mathbb{R}$ measurable in $\Omega$ for all $\left(t_{1}, \ldots, t_{n}\right) \in \mathbb{R}^{n}$ and $C^{1}$ in $\mathbb{R}^{n}$ for every $x \in \Omega$ satisfying $(\mathcal{G})$, there is a $\delta>0$, such that, for each $\mu \in[0, \delta]$ the system $\left(P_{1}\right)$ admits at least three weak solutions in $\left(W^{2, p_{1}}(\Omega) \cap W_{0}^{1, p_{1}}(\Omega)\right) \times \cdots \times\left(W^{2, p_{n}}(\Omega) \cap W_{0}^{1, p_{n}}(\Omega)\right)$ whose norms are less than $\rho$. Our main result is Theorem 1.1, which provides intervals for 
the parameters such that if the parameters belong to those intervals, the corresponding system has at least three solutions satisfying some boundedness properties. Our results generalize and unifies the results in [14].

Here in the sequel, $X$ will be denoted the Sobolev space $W^{2, p_{1}}(\Omega) \cap W_{0}^{1, p_{1}}(\Omega)$ and $E$ will be denoted the Cartesian product of Sobolev spaces $W^{2, p_{1}}(\Omega) \cap$ $W_{0}^{1, p_{1}}(\Omega), \ldots, W^{2, p_{n}}(\Omega) \cap W_{0}^{1, p_{n}}(\Omega)$, i.e., $E=\left(W^{2, p_{1}}(\Omega) \cap W_{0}^{1, p_{1}}(\Omega)\right) \times \cdots \times$ $\left(W^{2, p_{n}}(\Omega) \cap W_{0}^{1, p_{n}}(\Omega)\right)$. The space $E$ will be endowed with the norm $\left\|\left(u_{1}, \ldots, u_{n}\right)\right\|=\left\|u_{1}\right\|_{p_{1}}+\cdots+\left\|u_{n}\right\|_{p_{n}},\left\|u_{i}\right\|_{p_{i}}=\left(\int_{\Omega}\left|\Delta u_{i}\right|^{p_{i}} d x\right)^{\frac{1}{p_{i}}}, 1 \leq i \leq n$.

Let

$$
K=\max \left\{\sup _{u \in W^{2, p_{i}}(\Omega) \cap W_{0}^{1, p_{i}}(\Omega) \backslash\{0\}} \frac{\sup _{x \in \Omega}|u(x)|^{p_{i}}}{\|u\|_{p_{i}}^{p_{i}}}\right\}, 1 \leq i \leq n .
$$

Since $p_{i}>\max \left\{1, \frac{N}{2}\right\}, W^{2, p_{i}}(\Omega) \cap W_{0}^{1, p_{i}}(\Omega) \hookrightarrow C^{0}(\bar{\Omega}), 1 \leq i \leq n$, are compact, and one has $K<+\infty$. As usual, a weak solution of problem $\left(P_{1}\right)$ is any $\left(u_{1}, \ldots, u_{n}\right) \in E$ such that

$$
\begin{aligned}
& -\sum_{i=1}^{n} \int_{\Omega}\left|\Delta u_{i}\right|^{p_{i}-2} \Delta u_{i} \Delta \xi_{i} d x \\
= & \sum_{i=1}^{n} \lambda \int_{\Omega} F_{u_{i}}\left(x, u_{1}, \ldots, u_{n}\right) \xi_{i} d x+\sum_{i=1}^{n} \mu \int_{\Omega} G_{u_{i}}\left(x, u_{1}, \ldots, u_{n}\right) \xi_{i} d x
\end{aligned}
$$

for every $\left(\xi_{1}, \ldots, \xi_{n}\right) \in E$.

Now, for every $x^{0} \in \Omega$ and choice $r_{1}, r_{2}$ with $r_{2}>r_{1}>0$, such that $B\left(x^{0}, r_{1}\right) \subset B\left(x^{0}, r_{2}\right) \subseteq \Omega$, where $B\left(x^{0}, r_{1}\right)$ denotes the ball with center at $x^{0}$ and radius of $r_{1}$, put

(3)

$$
\theta_{i}= \begin{cases}\frac{3 N}{\left(r_{2}-r_{1}\right)\left(r_{2}+r_{1}\right)}\left(\frac{K \pi^{\frac{N}{2}}\left(\left(r_{2}+r_{1}\right)^{N}-\left(2 r_{1}\right)^{N}\right)}{2^{N} \Gamma\left(1+\frac{N}{2}\right)}\right)^{\frac{1}{p_{i}}}, \quad N<\frac{4 r_{1}}{r_{2}-r_{1}}, 1 \leq i \leq n, \\ \frac{12 r_{1}}{\left(r_{2}-r_{1}\right)^{2}\left(r_{2}+r_{1}\right)}\left(\frac{K \pi^{\frac{N}{2}}\left(\left(r_{2}+r_{1}\right)^{N}-\left(2 r_{1}\right)^{N}\right)}{2^{N} \Gamma\left(1+\frac{N}{2}\right)}\right)^{\frac{1}{p_{i}}}, \quad N \geq \frac{4 r_{1}}{r_{2}-r_{1}},\end{cases}
$$

where $\Gamma(\cdot)$ is the Gamma function. Our main result gives the following theorems.

Theorem 1.1. Suppose that $r_{2}>r_{1}>0$, such that $B\left(x^{0}, r_{2}\right) \subset \Omega$ and assume that there exist $n+2$ positive constants $c, d, s_{i}$ for $1 \leq i \leq n$ with $s_{i}<p_{i}$, $\sum_{i=1}^{n} \frac{\left(d \theta_{i}\right)^{p_{i}}}{p_{i}}>\frac{c}{\prod_{i=1}^{n} p_{i}}$, and a negative function $\alpha \in L^{1}(\Omega)$ such that

$\left(j_{1}\right) F\left(x, t_{1}, \ldots, t_{n}\right) \leq 0$ for a.e. $x \in \Omega \backslash B\left(x^{0}, r_{1}\right)$ and all $\left(t_{1}, \ldots, t_{n}\right) \in$ $[0, d] \times \cdots \times[0, d]$ 
$\left(j_{2}\right) \quad m(\Omega) \sum_{i=1}^{n} \frac{\left(d \theta_{i}\right)^{p_{i}}}{p_{i}} \inf _{\left(x, t_{1}, \ldots, t_{n}\right) \in \Omega \times A} F\left(x, t_{1}, \ldots, t_{n}\right)$ $>\frac{c}{\prod_{i=1}^{n} p_{i}} \int_{B\left(x^{0}, r_{1}\right)} F(x, d, \ldots, d) d x$ where $A=\left\{\left(t_{1}, \ldots, t_{n}\right) \mid \sum_{i=1}^{n} \frac{\left|t_{i}\right|^{p_{i}}}{p_{i}} \leq \frac{c}{\prod_{i=1}^{n} p_{i}}\right\}$;

(j3) $F\left(x, t_{1}, \ldots, t_{n}\right) \geq \alpha(x)\left(1+\sum_{i=1}^{n}\left|t_{i}\right|^{s_{i}}\right)$ for a.e. $x \in \Omega$ and all $t_{i} \in$ $\mathbb{R}, 1 \leq i \leq n$.

Then there exist an open interval $\Lambda \subseteq[0,+\infty)$ and a positive real number $\rho$ with the following property: for each $\lambda \in \Lambda$ and for every Carathéodory functions $G_{t_{i}}: \Omega \times \mathbb{R}^{n} \mapsto \mathbb{R}$, satisfying $(\mathcal{G})$, there exists $\delta>0$ such that, for each $\mu \in[0, \delta]$, problem $\left(P_{1}\right)$ has at least three solutions whose norms in $E$ are less than $\rho$.

Let $f$ be a continuous function in $\Omega$ and $g_{i}$ be a $C^{1}$ function for $1 \leq i \leq n$ and

$$
F\left(x, u_{1}, \ldots, u_{n}\right)=f(x)\left(\prod_{i=1}^{n} g_{i}\left(u_{i}\right)\right)
$$

for each $\left(x, u_{1}, \ldots, u_{n}\right) \in \Omega \times \mathbb{R}^{n}$. More precisely, we consider the following problem

$$
\left\{\begin{aligned}
-\Delta\left(\left|\Delta u_{1}\right|^{p_{1}-2} \Delta u_{1}\right)=\lambda f(x) g_{1}^{\prime}\left(u_{1}\right)\left(\prod_{i=1,,_{i \neq 1}}^{n} g_{i}\left(u_{i}\right)\right) & \\
\quad+\mu G_{u_{1}}\left(x, u_{1}, \ldots, u_{n}\right), & \text { in } \Omega, \\
-\Delta\left(\left|\Delta u_{2}\right|^{p_{2}-2} \Delta u_{2}\right)=\lambda f(x) g_{2}^{\prime}\left(u_{2}\right)\left(\prod_{i=1,,_{i \neq 2}}^{n} g_{i}\left(u_{i}\right)\right) & \\
\quad+\mu G_{u_{2}}\left(x, u_{1}, \ldots, u_{n}\right), & \text { in } \Omega, \\
\cdots & \\
\left.-\Delta\left(\left|\Delta u_{n}\right|^{p_{n}-2} \Delta u_{n}\right)=\lambda f(x) g_{n}^{\prime}\left(u_{n}\right)\left(\prod_{i=1,,_{i \neq n}}^{n} g_{i}\left(u_{i}\right)\right)\right) & \text { in } \Omega, \\
\quad+\mu G_{u_{n}}\left(x, u_{1}, \ldots, u_{n}\right), & \text { on } \partial \Omega .
\end{aligned}\right.
$$

Then, by using Theorem 1.1, we have the following result:

Corollary 1.1. Assume that there exist $n+2$ positive constants $c, d$, and $s_{i}$ for $1 \leq i \leq n$ with $\sum_{i=1}^{n} \frac{\left(d \theta_{i}\right)^{p_{i}}}{p_{i}}>\frac{c}{\prod_{i=1}^{n} p_{i}}, s_{i}<p_{i}$ for $1 \leq i \leq n$ and a negative function $\alpha(x) \in L^{1}(\Omega)$ such that

$\left(k_{1}\right) f(x) \leq 0$ for each $x \in \Omega \backslash B\left(x^{0}, r_{1}\right)$ and $g_{i} \leq 0$ for $t_{i} \in[0, d], 1 \leq i \leq n$;

$\left(k_{2}\right) \quad m(\Omega) \sum_{i=1}^{n} \frac{\left(d \sigma_{i}\right)^{p_{i}}}{p_{i}} \inf _{\left(t_{1}, \ldots, t_{n}\right) \in A} f(x) \prod_{i-1}^{n} g_{i}\left(t_{i}\right)$

$>c \prod_{i=1}^{n} \frac{g_{i}(d)}{p_{i}} \int_{B\left(x^{0}, r_{1}\right)} f(x) d x$,

where $A=\left\{\left(t_{1}, \ldots, t_{n}\right) \mid \sum_{i=1}^{n} \frac{\left|t_{i}\right|^{p_{i}}}{p_{i}} \leq \frac{c}{\prod_{i=1}^{n} p_{i}}\right\}$;

$\left(k_{3}\right) f(x) \prod_{i=1}^{n} g_{i}\left(t_{i}\right) \geq \alpha(x)\left(1+\sum_{i=1}^{n}\left|t_{i}\right|^{s_{i}}\right)$ for all $t_{i} \in \mathbb{R}, 1 \leq i \leq n$ and a.e. $x \in \Omega$.

Then there exist an open interval $\Lambda \subseteq[0,+\infty)$ and a positive real number $\rho$ with the following property: for each $\lambda \in \Lambda$ and for every Carathéodory functions 
$G_{t_{i}}: \Omega \times \mathbb{R}^{n} \mapsto \mathbb{R}$ for $1 \leq i \leq n$, satisfying $(\mathcal{G})$, there exists $\delta>0$ such that, for each $\mu \in[0, \delta]$, problem $\left(P_{2}\right)$ has at least three solutions whose norms in $E$ are less than $\rho$.

Here is a remarkable consequence of Theorem 1.1. Consider the problem $\left(P_{3}\right)$

$$
\begin{cases}-\Delta\left(\left|\Delta u_{1}\right|^{p_{1}-2} \Delta u_{1}\right)=\lambda F_{u_{1}}\left(u_{1}, \ldots, u_{n}\right)+\mu G_{u_{1}}\left(x, u_{1}, \ldots, u_{n}\right), & \text { in } \Omega, \\ -\Delta\left(\left|\Delta u_{2}\right|^{p_{2}-2} \Delta u_{2}\right)=\lambda F_{u_{2}}\left(u_{1}, \ldots, u_{n}\right)+\mu G_{u_{2}}\left(x, u_{1}, \ldots, u_{n}\right), & \text { in } \Omega, \\ \cdots & \text { in } \Omega, \\ -\Delta\left(\left|\Delta u_{n}\right|^{p_{n}-2} \Delta u_{n}\right)=\lambda F_{u_{n}}\left(u_{1}, \ldots, u_{n}\right)+\mu G_{u_{n}}\left(x, u_{1}, \ldots, u_{n}\right), & \text { on } \partial \Omega . \\ u_{i}=\Delta u_{i}=0 \text { for } 1 \leq i \leq n, & \end{cases}
$$

Now we state another theorem.

Theorem 1.2. Let $F: \mathbb{R}^{n} \mapsto \mathbb{R}$ be a $C^{1}$ function and there exist $n+2$ positive constants $c, d, s_{i}$ for $1 \leq i \leq n$ and a negative constant a with $\sum_{i=1}^{n} \frac{\left(d \theta_{i}\right)^{p_{i}}}{p_{i}}>$ $\frac{c}{\prod_{i=1}^{n} p_{i}}, s_{i}<p_{i}$ for $1 \leq i \leq n$ such that

$\left(l_{1}\right) \quad F\left(t_{1}, \ldots, t_{n}\right) \leq 0$ for all $\left(t_{1}, \ldots, t_{n}\right) \in[0, d] \times \cdots \times[0, d] ;$
$\left(l_{2}\right) \quad m(\Omega) \sum_{i=1}^{n} \frac{\left(d \sigma_{i}\right)^{p_{i}}}{p_{i}} \inf _{\left(t_{1}, \ldots, t_{n}\right) \in A} F\left(t_{1}, \ldots, t_{n}\right)$
$>\frac{c r_{1}^{N} \pi^{\frac{N}{2}}}{\Gamma\left(1+\frac{N}{2}\right) \prod_{i=1}^{n} p_{i}} F(d, \ldots, d)$,
where $A=\left\{\left(t_{1}, \ldots, t_{n}\right) \mid \sum_{i=1}^{n} \frac{\left|t_{i}\right|^{p_{i}}}{p_{i}} \leq \frac{c}{\prod_{i=1}^{n} p_{i}}\right\} ;$
$\left(l_{3}\right) \quad F\left(t_{1}, \ldots, t_{n}\right) \geq a\left(1+\sum_{i=1}^{n}\left|t_{i}\right|^{s_{i}}\right)$ for all $t_{i} \in \mathbb{R}, 1 \leq i \leq n$.

Then there exist an open interval $\Lambda \subseteq[0,+\infty)$ and a positive real number $\rho$ with the following property: for each $\lambda \in \Lambda$ and for some Carathéodory functions $G_{t_{i}}: \Omega \times \mathbb{R}^{n} \mapsto \mathbb{R}$, satisfying $(\mathcal{G})$, there exists $\delta>0$ such that, for each $\mu \in[0, \delta]$, problem $\left(P_{3}\right)$ has at least three solutions whose norms in $E$ are less than $\rho$.

If $N=1$, we can get a better result than Theorem 1.2. For simplicity, fixing $\Omega=] 0,1\left[, p_{i}>1\right.$, put

$$
k=\max \left\{\frac{1}{2^{p_{i}}} p_{i}^{-1}, i=1, \ldots, n\right\}
$$

then we have the following result.

Theorem 1.3. Let $F: \mathbb{R}^{n} \mapsto \mathbb{R}$ be a $C^{1}$ function and assume that there exist $n+2$ positive constants $c, d, s_{i}$ and a negative constant a with $\sum_{i=1}^{n} \frac{(32 d)^{p_{i}}}{2 K p_{i}}>$ $\frac{c}{\prod_{i=1}^{n} p_{i}}$ for $1 \leq i \leq n$, where $k$ is given by (4), such that

$\left(m_{1}\right) F\left(t_{1}, \ldots, t_{n}\right) \leq 0$ for all $\left(t_{1}, \ldots, t_{n}\right) \in[0, d] \times \cdots \times[0, d]$;

$\left(m_{2}\right) \sum_{i=1}^{n} \frac{(32 d)^{p_{i}}}{2 k p_{i}} \inf _{\left(t_{1}, \ldots, t_{n}\right) \in A} F\left(t_{1}, \ldots, t_{n}\right)>\frac{c}{2 \prod_{i=1}^{n} p_{i}} F(d, \ldots, d)$, where $A=\left\{\left(t_{1}, \ldots, t_{n}\right) \mid \sum_{i=1}^{n} \frac{\left|t_{i}\right|^{p_{i}}}{p_{i}} \leq \frac{c}{\prod_{i=1}^{n} p_{i}}\right\}$;

$\left(m_{3}\right) F\left(t_{1}, \ldots, t_{n}\right) \geq a\left(1+\sum_{i=1}^{n}\left|t_{i}\right|^{s_{i}}\right)$ for all $t_{i} \in \mathbb{R}, 1 \leq i \leq n$. 
Then there exist an open interval $\Lambda \subseteq[0,+\infty)$ and a positive real number $\rho$ with the following property: for each $\lambda \in \Lambda$ and for every Carathéodory functions $G_{t_{i}}: \Omega \times \mathbb{R}^{n} \mapsto \mathbb{R}$, satisfying $(\mathcal{G})$, there exists $\delta>0$ such that, for each $\mu \in[0, \delta]$, problem

$\left(P_{4}\right)$

$$
\begin{cases}-\left(\left|u_{1}^{\prime \prime}\right|^{p_{1}-2} u_{1}^{\prime \prime}\right)^{\prime \prime}=\lambda F_{u_{1}}\left(u_{1}, \ldots, u_{n}\right)+\mu G_{u_{1}}\left(x, u_{1}, \ldots, u_{n}\right), & \text { in }] 0,1[, \\ -\left(\left|u_{2}^{\prime \prime}\right|^{p_{2}-2} u_{2}^{\prime \prime}\right)^{\prime \prime}=\lambda F_{u_{2}}\left(u_{1}, \ldots, u_{n}\right)+\mu G_{u_{2}}\left(x, u_{1}, \ldots, u_{n}\right), & \text { in }] 0,1[, \\ \cdots & \\ -\left(\left|u_{n}^{\prime \prime}\right|^{p_{n}-2} u_{n}^{\prime \prime}\right)^{\prime \prime}=\lambda F_{u_{n}}\left(u_{1}, \ldots, u_{n}\right)+\mu G_{u_{n}}\left(x, u_{1}, \ldots, u_{n}\right), & \text { in }] 0,1[ \\ u_{i}(0)-u_{i}(1)=u_{i}^{\prime \prime}(0)-u_{i}^{\prime \prime}(1)=0 & \text { for } 1 \leq i \leq n,\end{cases}
$$

has at least three solutions whose norms in $W^{2, p_{1}}(0,1) \cap W_{0}^{1, p_{1}}(0,1) \times \cdots \times$ $W^{2, p_{n}}(0,1) \cap W_{0}^{1, p_{n}}(0,1)$ are less than $\rho$.

\section{Proof of theorems}

Our analysis is based on the following three critical points theorem to transfer the existence of three solutions of the system $\left(P_{1}\right)$ into the existence of critical points of the Euler functional.

Theorem 2.1 ([19], Theorem 1). Let $X$ be a reflexive real Banach space. $\Phi: X$ $\mapsto \mathbb{R}$ is a continuously Gâteaux differentiable and sequentially weakly lower semicontinuous functional whose Gâteaux derivative admits a continuous inverse on $X^{*}$ and $\Phi$ is bounded on each bounded subset of $X ; \Psi: X \mapsto \mathbb{R}$ is a continuously Gâteaux differentiable functional whose Gâteaux derivative is compact; $I \subseteq \mathbb{R}$ an interval. Assume that

$$
\lim _{\|x\| \rightarrow+\infty}(\Phi(x)+\lambda \Psi(x))=+\infty
$$

for all $\lambda \in I$, and that there exists $h \in \mathbb{R}$ such that

$$
\sup _{\lambda \in I} \inf _{x \in X}(\Phi(x)+\lambda(\Psi(x)+h))<\inf _{x \in X} \sup _{\lambda \in I}(\Phi(x)+\lambda(\Psi(x)+h)) .
$$

Then, there exists an open interval $\Lambda \subseteq I$ and a positive real number $\rho$ with the following property: for every $\lambda \in \Lambda$ and every $C^{1}$ functional $J: X \mapsto \mathbb{R}$ with compact derivative, there exists $\delta>0$ such that, for each $\mu \in[0, \delta]$ the equation

$$
\Phi^{\prime}(x)+\lambda \Psi^{\prime}(x)+\mu J^{\prime}(x)=0
$$

has at least three solutions in $X$ whose norms are less than $\rho$.

For using later, we also recall the following result, Proposition 3.1 of [18].

Proposition 2.1 ([18], Proposition 3.1). Let $X$ be a non-empty set and $\Phi, \Psi$ two real functions on $X$. Assume that there are $r>0$ and $x_{0}, x_{1} \in X$ such that

$$
\Phi\left(x_{0}\right)=\Psi\left(x_{0}\right)=0, \quad \Phi\left(x_{1}\right)>r, \quad \inf _{\left.\left.x \in \Phi^{-1}(]-\infty, r\right]\right)} \Psi(x)>r \frac{\Psi\left(x_{1}\right)}{\Phi\left(x_{1}\right)} .
$$


Then, for each $h$ satisfying

$$
\inf _{\left.\left.x \in \Phi^{-1}(]-\infty, r\right]\right)} \Psi(x)>h>r \frac{\Psi\left(x_{1}\right)}{\Phi\left(x_{1}\right)},
$$

one has

$$
\sup _{\lambda \geq 0} \inf _{x \in X}(\Phi(x)+\lambda(h+\Psi(x)))<\inf _{x \in X} \sup _{\lambda \geq 0}(\Phi(x)+\lambda(h+\Psi(x))) .
$$

Before giving the proof of Theorem 1.1, let us see the following two lemmas.

Lemma 2.1. Assume that there exist two positive constants $c, d$ with $\sum_{i=1}^{n} \frac{\left(d \theta_{i}\right)^{p_{i}}}{p_{i}}>\frac{c}{\prod_{i=1}^{n} p_{i}}$, such that

$\left(j_{1}\right) F\left(x, t_{1}, \ldots, t_{n}\right) \leq 0$ for a.e. $x \in \Omega \backslash B\left(x^{0}, r_{1}\right)$ and all $\left(t_{1}, \ldots, t_{n}\right) \in$ $[0, d] \times \cdots \times[0, d]$

$\left(j_{2}\right) \quad m(\Omega) \sum_{i=1}^{n} \frac{\left(d \theta_{i}\right)^{p_{i}}}{p_{i}} \inf _{\left(x, t_{1}, \ldots, t_{n}\right) \in \Omega \times A} F\left(x, t_{1}, \ldots, t_{n}\right)$ $>\frac{c}{\prod_{i=1}^{n} p_{i}} \int_{B\left(x^{0}, r_{1}\right)} F(x, d, \ldots, d) d x$

$$
\text { where } A=\left\{\left(t_{1}, \ldots, t_{n}\right) \mid \sum_{i=1}^{n} \frac{\left|t_{i}\right|^{p_{i}}}{p_{i}} \leq \frac{c}{\prod_{i=1}^{n} p_{i}}\right\} \text {; }
$$

Then there exist $u_{i}^{*} \in W^{2, p_{i}}(\Omega) \cap W_{0}^{1, p_{i}}(\Omega)$ for $1 \leq i \leq n$, such that

$$
\sum_{i=1}^{n} \frac{\left\|u_{i}^{*}\right\|_{p_{i}}^{p_{i}}}{p_{i}}>\frac{1}{\prod_{i=1}^{n} p_{i}} \frac{c}{K}
$$

and

$$
m(\Omega) \inf _{\left(x, t_{1}, \ldots, t_{n}\right) \in \Omega \times A} F\left(x, t_{1}, \ldots, t_{n}\right)>\frac{c}{K} \frac{\int_{\Omega} F\left(x, u_{1}^{*}(x), \ldots, u_{n}^{*}(x)\right) d x}{\sum_{i=1}^{n} \prod_{j=1, j \neq i}^{n} p_{j}\left\|u_{i}^{*}\right\|_{p_{i}}^{p_{i}}},
$$

where $A=\left\{\left(t_{1}, \ldots, t_{n}\right) \mid \sum_{i=1}^{n} \frac{\left|t_{i}\right|^{p_{i}}}{p_{i}} \leq \frac{c}{\prod_{i=1}^{n} p_{i}}\right\}$.

Proof. Let

(6)

$$
w(x)= \begin{cases}0, & x \in \Omega \backslash B\left(x^{0}, r_{2}\right), \\ \frac{d\left(3\left(l^{4}-r_{2}^{4}\right)-4\left(r_{1}+r_{2}\right)\left(l^{3}-r_{2}^{3}\right)+6 r_{1} r_{2}\left(l^{2}-r_{2}^{2}\right)\right)}{\left(r_{2}-r_{1}\right)^{3}\left(r_{1}+r_{2}\right)}, & x \in B\left(x^{0}, r_{2}\right) \backslash B\left(x^{0}, r_{1}\right), \\ d, & x \in B\left(x^{0}, r_{1}\right),\end{cases}
$$

where $u_{i}^{*}(x)=w(x)$ for $1 \leq i \leq n$ and $l=\operatorname{dist}\left(x, x^{0}\right)=\sqrt{\sum_{i=1}^{N}\left(x_{i}-x_{i}^{0}\right)^{2}}$.

We have

$$
\begin{aligned}
& \frac{\partial u_{i}^{*}(x)}{\partial x_{i}}= \begin{cases}0, & x \in \Omega \backslash B\left(x^{0}, r_{2}\right) \cup B\left(x^{0}, r_{1}\right), \\
\frac{12 d\left(l^{2}\left(x_{i}-x_{i}^{0}\right)-\left(r_{1}+r_{2}\right) l\left(x_{i}-x_{i}^{0}\right)+r_{1} r_{2}\left(x_{i}-x_{i}^{0}\right)\right)}{\left(r_{2}-r_{1}\right)^{3}\left(r_{1}+r_{2}\right)}, & x \in B\left(x^{0}, r_{2}\right) \backslash B\left(x^{0}, r_{1}\right),\end{cases} \\
& \frac{\partial^{2} u_{i}^{*}(x)}{\partial x_{i}^{2}}= \begin{cases}0, & x \in \Omega \backslash B\left(x^{0}, r_{2}\right) \cup B\left(x^{0}, r_{1}\right), \\
\frac{12 d\left(r_{1} r_{2}+\left(2 l-r_{1}-r_{2}\right)\left(x_{i}-x_{i}^{0}\right)^{2} / l-\left(r_{2}+r_{1}-l\right) l\right)}{\left(r_{2}-r_{1}\right)^{3}\left(r_{1}+r_{2}\right)}, & x \in B\left(x^{0}, r_{2}\right) \backslash B\left(x^{0}, r_{1}\right),\end{cases}
\end{aligned}
$$




$$
\sum_{i=1}^{N} \frac{\partial^{2} u_{i}^{*}(x)}{\partial x_{i}^{2}}= \begin{cases}0, & x \in \Omega \backslash B\left(x^{0}, r_{2}\right) \cup B\left(x^{0}, r_{1}\right), \\ \frac{12 d\left((N+2) l^{2}-(N+1)\left(r_{1}+r_{2}\right) l+N r_{1} r_{2}\right)}{\left(r_{2}-r_{1}\right)^{3}\left(r_{1}+r_{2}\right)}, & x \in B\left(x^{0}, r_{2}\right) \backslash B\left(x^{0}, r_{1}\right) .\end{cases}
$$

It is easy to verify that $u_{i}^{*} \in W^{2, p_{i}}(\Omega) \cap W_{0}^{1, p_{i}}(\Omega)$, and, in particular, one has

$$
\begin{aligned}
\left\|u_{i}^{*}\right\|_{p_{i}}^{p_{i}}= & \frac{(12 d)^{p_{i}} 2 \pi^{\frac{N}{2}}}{\left(r_{2}-r_{1}\right)^{3 p_{i}}\left(r_{1}+r_{2}\right)^{p_{i}} \Gamma\left(\frac{N}{2}\right)} \\
& \times \int_{r_{1}}^{r_{2}}\left|(N+2) r^{2}-(N+1)\left(r_{1}+r_{2}\right) r+N r_{1} r_{2}\right|^{p_{i}} r^{N-1} d r .
\end{aligned}
$$

Here, we obtain from (3) and (7) that

$$
\frac{\theta_{i}^{p_{i}} d^{p_{i}}}{K}<\left\|u_{i}^{*}\right\|_{p_{i}}^{p_{i}} \text { for } 1 \leq i \leq n
$$

By the assumption

$$
\sum_{i=1}^{n} \frac{\left(d \theta_{i}\right)^{p_{i}}}{p_{i}}>\frac{c}{\prod_{i=1}^{n} p_{i}}
$$

it follows from (8) that

$$
\sum_{i=1}^{n} \frac{\left\|u_{i}^{*}\right\|_{p_{i}}^{p_{i}}}{p_{i}}>\frac{1}{K}\left(\sum_{i=1}^{n} \frac{d^{p_{i}} \theta_{i}^{p_{i}}}{p_{i}}\right)>\frac{1}{\prod_{i=1}^{n} p_{i}} \frac{c}{K} .
$$

Since $0 \leq u_{i}^{*} \leq d$ for each $x \in \Omega, 1 \leq i \leq n$, the condition $\left(j_{1}\right)$ ensures that

$$
\begin{aligned}
& \int_{\Omega \backslash B\left(x^{0}, r_{2}\right)} F\left(x, u_{1}^{*}(x), \ldots, u_{n}^{*}(x)\right) d x \\
& +\int_{B\left(x^{0}, r_{2}\right) \backslash B\left(x^{0}, r_{1}\right)} F\left(x, u_{1}^{*}(x), \ldots, u_{n}^{*}(x)\right) d x \leq 0 .
\end{aligned}
$$

Hence, by condition $\left(j_{2}\right)$ and $(8)$, we have

$$
\begin{aligned}
& m(\Omega)_{\left(x, t_{1}, \ldots, t_{n}\right) \in \Omega \times A} F\left(x, t_{1}, \ldots, t_{n}\right) \\
> & \frac{c}{\prod_{i=1}^{n} p_{i}\left(\frac{\sum_{i=1}^{n} d^{p_{i}} \theta_{i}^{p_{i}}}{p_{i}}\right)} \int_{B\left(x^{0}, r_{1}\right)} F(x, d, \ldots, d) d x \\
> & \frac{c}{K \prod_{i=1}^{n} p_{i}} \frac{1}{\sum_{i=1}^{n} \frac{\left\|u_{i}^{*}\right\|_{p_{i}}^{p_{i}}}{p_{i}}} \int_{B\left(x^{0}, r_{1}\right)} F(x, d, \ldots, d) d x \\
\geq & \frac{c}{K} \frac{\int_{\Omega} F\left(x, u_{1}^{*}(x), \ldots, u_{n}^{*}(x)\right) d x}{\sum_{i=1}^{n} \prod_{j=1, j \neq i}^{n} p_{j}\left\|u_{i}^{*}\right\|_{p_{i}}^{p_{i}}} .
\end{aligned}
$$

Lemma 2.2. Let $T: E \mapsto E^{*}$ be the operator defined by

$$
\left\langle T\left(u_{1}, \ldots, u_{n}\right),\left(\xi_{1}, \ldots, \xi_{n}\right)\right\rangle=\sum_{i=1}^{n} \int_{\Omega}\left|\Delta u_{i}(x)\right|^{p_{i}-2} \Delta u_{i}(x) \Delta \xi_{i}(x) d x
$$


for all $\left(u_{1}, \ldots, u_{n}\right),\left(\xi_{1}, \ldots, \xi_{n}\right) \in E$, where $E^{*}$ denotes the dual of $E$. Then $T$ admits a continuous inverse on $E^{*}$.

Proof. Taking into account (2.2) of [20] for $p \geq 2$, there exists a positive constant $c_{p}$ such that

$$
\left\langle|x|^{p-2} x-|y|^{p-2} y, x-y\right\rangle \geq c_{p}|x-y|^{p},
$$

where $\langle\cdot, \cdot\rangle$ denotes the usual inner product in $\mathbb{R}^{N}$ for every $x, y \in \mathbb{R}^{N}$. Thus, it is easy to see that

$$
\begin{aligned}
& \left(T\left(u_{1}, \ldots, u_{n}\right)-T\left(v_{1}, \ldots, v_{n}\right)\right)\left(u_{1}-v_{1}, \ldots, u_{n}-v_{n}\right) \\
\geq & \min \left\{c_{p_{1}}, \ldots, c_{p_{n}}\right\} \sum_{i=1}^{n}\left\|u_{i}-v_{i}\right\|_{p_{i}}^{p_{i}}
\end{aligned}
$$

for every $\left(u_{1}, \ldots, u_{n}\right),\left(v_{1}, \ldots, v_{n}\right) \in E$, which means that $T$ is uniformly monotone. Therefore, since $T$ is coercive and hemicontinuous in $X$ (for more details, see [14, Lemma 2]), by applying Theorem 26.A of [22], we have that $T$ admits a continuous inverse on $E^{*}$.

Now we can give the proof of our main results.

Proof of Theorem 1.1. For each $\left(u_{1}, \ldots, u_{n}\right) \in E$, let

$$
\begin{gathered}
\Phi\left(u_{1}, \ldots, u_{n}\right)=\sum_{i=1}^{n} \frac{\left\|u_{i}\right\|_{p_{i}}^{p_{i}}}{p_{i}} \\
\Psi\left(u_{1}, \ldots, u_{n}\right)=\int_{\Omega} F\left(x, u_{1}, \ldots, u_{n}\right) d x
\end{gathered}
$$

and

$$
J\left(u_{1}, \ldots, u_{n}\right)=\int_{\Omega} G\left(x, u_{1}, \ldots, u_{n}\right) d x .
$$

Under the condition of Theorem 1.1, $\Phi$ is a continuously Gâteaux differentiable and sequentially weakly lower semicontinuous functional. Moreover, from Lemma 2.2 the Gâteaux derivative of $\Phi$ admits a continuous inverse on $E^{*}$. $\Psi$ and $J$ are continuously Gâteaux differential functionals whose Gâteaux derivatives are compact. Obviously, $\Phi$ is bounded on each bounded subset of $E$. In particular, for each $\left(u_{1}, \ldots, u_{n}\right),\left(\xi_{1}, \ldots, \xi_{n}\right) \in E$,

$$
\begin{aligned}
\left\langle\Phi^{\prime}\left(u_{1}, \ldots, u_{n}\right),\left(\xi_{1}, \ldots, \xi_{n}\right)\right\rangle & =\sum_{i=1}^{n} \int_{\Omega}\left|\Delta u_{i}\right|^{p_{i}-2} \Delta u_{i} \Delta \xi_{i} d x \\
\left\langle\Psi^{\prime}\left(u_{1}, \ldots, u_{n}\right),\left(\xi_{1}, \ldots, \xi_{n}\right)\right\rangle & =\sum_{i=1}^{n} \lambda \int_{\Omega} F_{u_{i}}\left(x, u_{1}, \ldots, u_{n}\right) \xi_{i} d x \\
\left\langle J^{\prime}\left(u_{1}, \ldots, u_{n}\right),\left(\xi_{1}, \ldots, \xi_{n}\right)\right\rangle & =\sum_{i=1}^{n} \mu \int_{\Omega} G_{u_{i}}\left(x, u_{1}, \ldots, u_{n}\right) \xi_{i} d x .
\end{aligned}
$$


Hence, it follows from $(2)$ that the weak solutions of systems $\left(P_{1}\right)$ are exactly the solutions of the equation

$$
\Phi^{\prime}\left(u_{1}, \ldots, u_{n}\right)+\lambda \Psi^{\prime}\left(u_{1}, \ldots, u_{n}\right)+\mu J^{\prime}\left(u_{1}, \ldots, u_{n}\right)=0 .
$$

Thanks to $\left(j_{3}\right)$, for each $\lambda>0$, one has that

$$
\lim _{\left\|\left(u_{1}, \ldots, u_{n}\right)\right\| \rightarrow+\infty}\left(\Phi\left(u_{1}, \ldots, u_{n}\right)+\lambda \Psi\left(u_{1}, \ldots, u_{n}\right)\right)=+\infty,
$$

and so the first assumption of Theorem 2.1 holds.

Thanks to Lemma 2.1 , there exists $\left(u_{1}^{*}, \ldots, u_{n}^{*}\right) \in E$ such that

$$
\Phi\left(u_{1}^{*}, \ldots, u_{n}^{*}\right)=\sum_{i=1}^{n} \frac{\left\|u_{i}^{*}\right\|_{p_{i}}^{p_{i}}}{p_{i}}>\frac{1}{\prod_{i=1}^{n} p_{i}} \frac{c}{K}>0=\Phi(0, \ldots, 0)
$$

and

(11) $m(\Omega) \inf _{\left(x, t_{1}, \ldots, t_{n}\right) \in \Omega \times A} F\left(x, t_{1}, \ldots, t_{n}\right)>\frac{c}{K} \frac{\int_{\Omega} F\left(x, u_{1}^{*}(x), \ldots, u_{n}^{*}(x)\right) d x}{\sum_{i=1}^{n} \prod_{j=1, j \neq i}^{n} p_{j}\left\|u_{i}^{*}\right\|_{p_{i}}^{p_{i}}}$,

where $A=\left\{\left(t_{1}, \ldots, t_{n}\right) \mid \sum_{i=1}^{n} \frac{\left|t_{i}\right|^{p_{i}}}{p_{i}} \leq \frac{c}{\prod_{i=1}^{n} p_{i}}\right\}$.

Now, we obtain from (1) that

$$
\sup _{x \in \Omega}\left|u_{i}(x)\right|^{p_{i}} \leq K\left\|u_{i}\right\|_{p_{i}}^{p_{i}} \text { for } 1 \leq i \leq n,
$$

for each $\left(u_{1}, \ldots, u_{n}\right) \in E$, and then we have

$$
\sup _{x \in \Omega}\left\{\sum_{i=1}^{n} \frac{\left|u_{i}(x)\right|^{p_{i}}}{p_{i}}\right\} \leq K \sum_{i=1}^{n} \frac{\left\|u_{i}\right\|_{p_{i}}^{p_{i}}}{p_{i}}
$$

for each $\left(u_{1}, \ldots, u_{n}\right) \in E$. Let $r=\frac{1}{\prod_{i=1}^{n} p_{i}} \frac{c}{K}$, for each $\left(u_{1}, \ldots, u_{n}\right) \in E$ such that

$$
\Phi\left(u_{1}, \ldots, u_{n}\right)=\sum_{i=1}^{n} \frac{\left\|u_{i}\right\|_{p_{i}}^{p_{i}}}{p_{i}} \leq r
$$

by (12) one has

$$
\sup _{x \in \Omega}\left\{\sum_{i=1}^{n} \frac{\left|u_{i}(x)\right|^{p_{i}}}{p_{i}}\right\} \leq \frac{c}{\prod_{i=1}^{n} p_{i}}
$$

So, it follows from (13) and (11) that

$$
\begin{aligned}
& \inf _{\left\{\left(u_{1}, \ldots, u_{n}\right) \mid \Phi\left(u_{1}, \ldots, u_{n}\right) \leq r\right\}}\left(\Psi\left(u_{1}, \ldots, u_{n}\right)\right) \\
= & \left\{\inf _{\left(u_{1}, \ldots, u_{n}\right) \mid \sum_{i=1}^{n} \frac{\left\|u_{i}\right\|_{p_{i}}^{p_{i}}}{p_{i}} \leq r} F\left(x, u_{1}, \ldots, u_{n}\right) d x\right. \\
\geq & \int_{\Omega} \inf _{\Omega} F\left(x, t_{1}, \ldots, t_{n}\right) d x
\end{aligned}
$$




$$
\begin{aligned}
& \geq m(\Omega) \inf _{\left(x, t_{1}, \ldots, t_{n}\right) \in \Omega \times A} F\left(x, t_{1}, \ldots, t_{n}\right) \\
& >\frac{c}{K \prod_{i=1}^{n} p_{i}} \frac{\int_{\Omega} F\left(x, u_{1}^{*}(x), \ldots, u_{n}^{*}(x)\right) d x}{\sum_{i=1}^{n} \frac{\left\|u_{i}^{*}\right\|_{p_{i}}^{p_{i}}}{p_{i}}} \\
& =r \frac{\int_{\Omega} F\left(x, u_{1}^{*}(x), \ldots, u_{n}^{*}(x)\right) d x}{\sum_{i=1}^{n} \frac{\left\|u_{i}^{*}\right\|_{p_{i}}^{p_{i}}}{p_{i}}} \\
& =r \frac{\Psi\left(u_{1}^{*}, \ldots, u_{n}^{*}\right)}{\Phi\left(u_{1}^{*}, \ldots, u_{n}^{*}\right)}
\end{aligned}
$$

So, one has

$$
\inf _{\left\{\left(u_{1}, \ldots, u_{n}\right) \mid \Phi\left(u_{1}, \ldots, u_{n}\right) \leq r\right\}}\left(\Psi\left(u_{1}, \ldots, u_{n}\right)\right)>r \frac{\Psi\left(u_{1}^{*}, \ldots, u_{n}^{*}\right)}{\Phi\left(u_{1}^{*}, \ldots, u_{n}^{*}\right)} .
$$

Fixing $h$ such that

$$
\inf _{\left\{\left(u_{1}, \ldots, u_{n}\right) \mid \Phi\left(u_{1}, \ldots, u_{n}\right) \leq r\right\}}\left(\Psi\left(u_{1}, \ldots, u_{n}\right)\right)>h>r \frac{\Psi\left(u_{1}^{*}, \ldots, u_{n}^{*}\right)}{\Phi\left(u_{1}^{*}, \ldots, u_{n}^{*}\right)},
$$

from (10), (14) and Proposition 1, with $\left(u_{0_{1}}, \ldots, u_{0_{n}}\right)=(0, \ldots, 0)$ and $\left(u_{1_{1}}, \ldots\right.$, $\left.u_{1_{n}}\right)=\left(u_{1}^{*}, \ldots, u_{n}^{*}\right)$, we obtain

$$
\sup _{\lambda \geq 0} \inf _{x \in X}(\Phi(x)+\lambda(h+\Psi(x)))<\inf _{x \in X} \sup _{\lambda \geq 0}(\Phi(x)+\lambda(h+\Psi(x))),
$$

and so the assumption (5) of Theorem 2.1 holds.

Now, all assumptions of Theorem 2.1 are satisfied. Hence, applying Theorem 2.1 , and taking into account that the critical points of the functional $\Phi+\lambda \Psi+\mu J$ are exactly the weak solutions of the system $\left(P_{1}\right)$, we have the conclusion.

Proof of Theorem 1.2. From $\left(l_{2}\right)$ and since

we have

$$
\int_{B\left(x^{0}, r_{1}\right)} F(d, \ldots, d) d x=r_{1}^{N} \frac{\pi^{\frac{N}{2}}}{\Gamma\left(1+\frac{N}{2}\right)} F(d, \ldots, d),
$$

$$
m(\Omega) \sum_{i=1}^{n} \frac{\left(d \theta_{i}\right)^{p_{i}}}{p_{i}} \inf _{\left(t_{1}, \ldots, t_{n}\right) \in A} F\left(t_{1}, \ldots, t_{n}\right)>\frac{c}{\prod_{i=1}^{n} p_{i}} \int_{B\left(x^{0}, r_{1}\right)} F(d, \ldots, d) d x .
$$

So, we have the conclusion by Theorem 1.1.

Proof of Theorem 1.3. For each $\left(u_{1}, \ldots, u_{n}\right) \in W^{2, p_{1}}(0,1) \cap W_{0}^{1, p_{1}}(0,1) \times \cdots \times$ $W^{2, p_{n}}(0,1) \cap W_{0}^{1, p_{n}}(0,1)$, let

$$
\begin{aligned}
& \Phi\left(u_{1}, \ldots, u_{n}\right)=\sum_{i=1}^{n} \frac{\left\|u_{i}\right\|_{p_{i}}^{p_{i}}}{p_{i}} \\
& \Psi\left(u_{1}, \ldots, u_{n}\right)=\int_{0}^{1} F\left(u_{1}, \ldots, u_{n}\right) d x,
\end{aligned}
$$




$$
J\left(u_{1}, \ldots, u_{n}\right)=\int_{\Omega} G\left(x, u_{1}, \ldots, u_{n}\right) d x .
$$

Under the condition, $\Phi$ is a continuously Gâteaux differentiable and sequentially weakly lower semicontinuous functional. Moreover, from Lemma 2.2 the Gâteaux derivative of $\Phi$ admits a continuous inverse on $X^{*} . \Psi$ and $J$ are continuously Gâteaux differential functionals whose Gâteaux derivatives are compact. Obviously, $\Phi$ is bounded on each bounded subset of $W^{2, p_{1}}(0,1) \cap$ $W_{0}^{1, p_{1}}(0,1) \times \cdots \times W^{2, p_{n}}(0,1) \cap W_{0}^{1, p_{n}}(0,1)$. Hence, it is well known that the weak solutions of systems are exactly the solutions of the equation

$$
\Phi^{\prime}\left(u_{1}, \ldots, u_{n}\right)+\lambda \Psi^{\prime}\left(u_{1}, \ldots, u_{n}\right)+\mu J^{\prime}\left(u_{1}, \ldots, u_{n}\right)=0 .
$$

Thanks to $\left(m_{3}\right)$, for each $\lambda>0$, one has that

$$
\lim _{\left\|\left(u_{1}, \ldots, u_{n}\right)\right\| \rightarrow+\infty}\left(\Phi\left(u_{1}, \ldots, u_{n}\right)+\lambda \Psi\left(u_{1}, \ldots, u_{n}\right)\right)=+\infty,
$$

and so the first assumption of Theorem 2.1 holds.

Now, let us consider

$$
r=\frac{1}{\prod_{i=1}^{n} p_{i}} \frac{c}{K}
$$

We obtain from (1) that

$$
\sup _{x \in(0,1)}\left|u_{i}(x)\right|^{p_{i}} \leq K\left\|u_{i}\right\|_{p_{i}}^{p_{i}} \text { for } 1 \leq i \leq n
$$

for each $\left(u_{1}, \ldots, u_{n}\right) \in W^{2, p_{1}}(0,1) \cap W_{0}^{1, p_{1}}(0,1) \times \cdots \times W^{2, p_{n}}(0,1) \cap W_{0}^{1, p_{n}}(0,1)$, and then we have

$$
\sup _{x \in(0,1)}\left\{\sum_{i=1}^{n} \frac{\left|u_{i}(x)\right|^{p_{i}}}{p_{i}}\right\} \leq K \sum_{i=1}^{n} \frac{\left\|u_{i}\right\|_{p_{i}}^{p_{i}}}{p_{i}}
$$

for each $\left(u_{1}, \ldots, u_{n}\right) \in W^{2, p_{1}}(0,1) \cap W_{0}^{1, p_{1}}(0,1) \times \cdots \times W^{2, p_{n}}(0,1) \cap W_{0}^{1, p_{n}}(0,1)$. Hence, for each $\left(u_{1}, \ldots, u_{n}\right) \in W^{2, p_{1}}(0,1) \cap W_{0}^{1, p_{1}}(0,1) \times \cdots \times W^{2, p_{n}}(0,1) \cap$ $W_{0}^{1, p_{n}}(0,1)$ such that

$$
\Phi\left(u_{1}, \ldots, u_{n}\right)=\sum_{i=1}^{n} \frac{\left\|u_{i}\right\|_{p_{i}}^{p_{i}}}{p_{i}} \leq r,
$$

by (17) one has

$$
\sup _{x \in(0,1)}\left\{\sum_{i=1}^{n} \frac{\left|u_{i}(x)\right|^{p_{i}}}{p_{i}}\right\} \leq \frac{c}{\prod_{i=1}^{n} p_{i}} .
$$

Now if we put $u_{i}^{*}(x)=w(x)$, where

$$
w(x)= \begin{cases}d-16 d\left(\frac{1}{4}-\left|x-\frac{1}{2}\right|\right)^{2}, & \left.\left.x \in\left[0, \frac{1}{4}\right] \cup\right] \frac{3}{4}, 1\right], \\ d, & \left.x \in] \frac{1}{4}, \frac{3}{4}\right],\end{cases}
$$


it is easy to verify that $\left(u_{1}^{*}, \ldots, u_{n}^{*}\right) \in W^{2, p_{1}}(0,1) \cap W_{0}^{1, p_{1}}(0,1) \times \cdots \times W^{2, p_{n}}(0,1)$ $\cap W_{0}^{1, p_{n}}(0,1)$ and get

$$
\left\|u_{i}^{*}\right\|_{p_{i}}^{p_{i}}=\frac{(32 d)^{p_{i}}}{2}
$$

Now, under the assumption of $\sum_{i=1}^{n} \frac{(32 d)^{p_{i}}}{2 K p_{i}}>\frac{c}{\prod_{i=1}^{n} p_{i}}$, we have

$$
\Phi\left(u_{1}^{*}, \ldots, u_{n}^{*}\right)=\sum_{i=1}^{n} \frac{\left\|u_{i}^{*}\right\|_{p_{i}}^{p_{i}}}{p_{i}}>\frac{1}{\prod_{i=1}^{n} p_{i}} \frac{c}{K}>0=\Phi(0, \ldots, 0) .
$$

Moreover, $0 \leq u_{i}^{*} \leq d$, it follows from $\left(m_{1}\right),\left(m_{2}\right)$ and $(20)$ that

$$
\begin{aligned}
\inf _{\left(t_{1}, \ldots, t_{n}\right) \in A} F\left(t_{1}, \ldots, t_{n}\right) & >\frac{c}{2 \prod_{i=1}^{n} p_{i}\left(\sum_{i=1}^{n} \frac{(32 d)^{p_{i}}}{2 K p_{i}}\right)} F(d, \ldots, d) \\
& >\frac{c}{K \prod_{i=1}^{n} p_{i}} \frac{1}{\sum_{i=1}^{n} \frac{\left\|u_{i}^{*}\right\|_{p_{i}}^{p_{i}}}{p_{i}}} \int_{0}^{1} F\left(u_{1}^{*}(x), \ldots, u_{n}^{*}(x)\right) d x \\
& \leq \frac{c}{K} \frac{\int_{0}^{1} F\left(u_{1}^{*}(x), \ldots, u_{n}^{*}(x)\right) d x}{\sum_{i=1}^{n} \prod_{j=1, j \neq i}^{n} p_{j}\left\|u_{i}^{*}\right\|_{p_{i}}^{p_{i}}}
\end{aligned}
$$

where $A=\left\{\left(t_{1}, \ldots, t_{n}\right) \mid \sum_{i=1}^{n} \frac{\left|t_{i}\right|^{p_{i}}}{p_{i}} \leq \frac{c}{\prod_{i=1}^{n} p_{i}}\right\}$.

So, it follows from (18) and (22) that

$$
\begin{aligned}
& \inf _{\left\{\left(u_{1}, \ldots, u_{n}\right) \mid \Phi\left(u_{1}, \ldots, u_{n}\right) \leq r\right\}}\left(\Psi\left(u_{1}, \ldots, u_{n}\right)\right) \\
= & \left\{\int_{\left.\left(u_{1}, \ldots, u_{n}\right) \mid \sum_{i=1}^{n} \frac{\left\|u_{i}\right\|_{p_{i}}^{p_{i}}}{p_{i}} \leq r\right\}}^{1} F\left(u_{1}, \ldots, u_{n}\right) d x\right. \\
\geq & \int_{0}^{1} \inf _{\left(t_{1}, \ldots, t_{n}\right) \in A} F\left(t_{1}, \ldots, t_{n}\right) d x \\
\geq & \inf _{\left(t_{1}, \ldots, t_{n}\right) \in A} F\left(t_{1}, \ldots, t_{n}\right) \\
> & \frac{c \quad \int_{0}^{1} F\left(u_{1}^{*}(x), \ldots, u_{n}^{*}(x)\right) d x}{K \prod_{i=1}^{n} p_{i}} \frac{\left\|u_{i}^{*}\right\|_{p_{i}}^{p_{i}}}{p_{i}} \\
= & r \frac{\int_{0}^{1} F\left(u_{1}^{*}(x), \ldots, u_{n}^{*}(x)\right) d x}{\sum_{i=1}^{n} \frac{\left\|u_{i}^{*}\right\|_{p_{i}}^{p_{i}}}{p_{i}}} \\
= & r \frac{\Psi\left(u_{1}^{*}, \ldots, u_{n}^{*}\right)}{\Phi\left(u_{1}^{*}, \ldots, u_{n}^{*}\right)} .
\end{aligned}
$$


Fixing $h$ such that

$$
\inf _{\left\{\left(u_{1}, \ldots, u_{n}\right) \mid \Phi\left(u_{1}, \ldots, u_{n}\right) \leq r\right\}}\left(\Psi\left(u_{1}, \ldots, u_{n}\right)\right)>h>r \frac{\Psi\left(u_{1}^{*}, \ldots, u_{n}^{*}\right)}{\Phi\left(u_{1}^{*}, \ldots, u_{n}^{*}\right)},
$$

from $(21),(23)$ and Proposition 1 , with $\left(u_{0_{1}}, \ldots, u_{0_{n}}\right)=(0, \ldots, 0)$ and $\left(u_{1_{1}}, \ldots\right.$, $\left.u_{1_{n}}\right)=\left(u_{1}^{*}, \ldots, u_{n}^{*}\right)$, we obtain

$$
\sup _{\lambda \geq 0} \inf _{x \in X}(\Phi(x)+\lambda(h+\Psi(x)))<\inf _{x \in X} \sup _{\lambda \geq 0}(\Phi(x)+\lambda(h+\Psi(x))),
$$

and so the assumption (5) of Theorem 2.1 holds.

Now, all assumptions of Theorem 2.1 are satisfied. Hence, applying Theorem 2.1 , we have the conclusion.

Acknowledgments. The author would like to thank reviewers for clear valuable comments and suggestions.

\section{References}

[1] G. A. Afrouzi and S. Heidarkhani, Existence of three solutions for a class of Dirichlet quasilinear elliptic systems involving the $\left(p_{1}, \ldots, p_{n}\right)$-Laplacian, Nonlinear Anal. 70 (2009), no. 1, 135-143.

[2] Multiplicity results for a two-point boundary value double eigenvalue problem, Ric. Mat. 59 (2010), no. 1, 39-47.

[3] _ Multiplicity theorems for a class of Dirichlet quasilinear elliptic systems involving the $\left(p_{1}, \ldots, p_{n}\right)$-Laplacian, Nonlinear Anal. 73 (2010), no. 8, 2594-2602.

[4] G. A. Afrouzi, S. Heidarkhani, and D. O'Regan, Three solutions to a class of Neumann doubly eigenvalue elliptic systems driven by a $\left(p_{1}, \ldots, p_{n}\right)$-Laplacian, Bull. Korean Math. Soc. 47 (2010), no. 6, 1235-1250.

[5] G. Bonanno and B. Di Bella. A boundary value problem for fourth-order elastic beam equations, J. Math. Anal. Appl. 343 (2008), no. 2, 1166-1176.

[6] F. Cammaroto, A. Chinnì, and B. Di Bella, Multiple solutions for a Neumann problem involving the $p(x)$-Laplacian, Nonlinear Anal. 71 (2009), no. 10, 4486-4492.

[7] J. Chabrowski and J. Marcos do Ó, On some fourth-order semilinear elliptic problems in $\mathbb{R}^{N}$, Nonlinear Anal. 49 (2002), no. 6, 861-884.

[8] S. El Manouni and M. Kbiri Alaoui, A result on elliptic systems with Neumann conditions via Ricceri's three critical points theorem, Nonlinear Anal. 71 (2009), no. 5-6, $2343-2348$.

[9] J. R. Graef, S. Heidarkhani, and L. Kong, A critical points approach to multiplicity results for multi-point boundary value problems, Appl. Anal. 90 (2011), no. 12, 19091925.

[10] S. Heidarkhani and Y. Tian, Multiplicity results for a class of gradient systems depending on two parameters, Nonlinear Anal. 73 (2010), no. 2, 547-554.

[11] _ . Three solutions for a class of gradient Kirchhoff-type systems depending on two parameters, Dynam. Systems Appl. 20 (2011), no. 4, 551-562.

[12] C. Li and C.-L. Tang, Three solutions for a class of quasilinear elliptic systems involving the $(p, q)$-Laplacian, Nonlinear Anal. 69 (2008), no. 10, 3322-3329.

[13] _. Three solutions for a Navier boundary value problem involving the p-biharmonic, Nonlinear Anal. 72 (2010), no. 3-4, 1339-1347.

[14] L. Li and C.-L. Tang, Existence of three solutions for $(p, q)$-biharmonic systems, Nonlinear Anal. 73 (2010), no. 3, 796-805. 
[15] J. Liu and X. Shi, Existence of three solutions for a class of quasilinear elliptic systems involving the $(p(x), q(x))$-Laplacian, Nonlinear Anal. 71 (2009), no. 1-2, 550-557.

[16] X.-L. Liu and W.-T. Li, Existence and multiplicity of solutions for fourth-order boundary value problems with parameters, J. Math. Anal. Appl. 327 (2007), no. 1, 362-375.

[17] A. M. Micheletti and A. Pistoia, Multiplicity results for a fourth-order semilinear elliptic problem, Nonlinear Anal. 31 (1998), no. 7, 895-908.

[18] B. Ricceri, Existence of three solutions for a class of elliptic eigenvalue problems, Math. Comput. Modelling 32 (2000), no. 11-13, 1485-1494.

[19] _ A three critical points theorem revisited, Nonlinear Anal. 70 (2009), no. 9, 3084-3089

[20] J. Simon, Régularité de la solution d'une équation non linéaire dans $\mathbb{R}^{N}$, In Journées d'Analyse Non Linéaire (Proc. Conf., Besançon, 1977), volume 665 of Lecture Notes in Math., pages 205-227, Springer, Berlin, 1978.

[21] J. Sun, H. Chen, J. J. Nieto, and M. Otero-Novoa, The multiplicity of solutions for perturbed second-order Hamiltonian systems with impulsive effects, Nonlinear Anal. 72 (2010), no. 12, 4575-4586.

[22] E. Zeidler, Nonlinear Functional Analysis and Its Applications. II/B, Nonlinear monotone operators. Translated from the German by the author and Leo F. Boron.SpringerVerlag, New York, 1990.

[23] L. Zhang and W. Ge, Solvability of a kind of Sturm-Liouville boundary value problems with impulses via variational methods, Acta Appl. Math. 110 (2010), no. 3, 1237-1248.

School of Mathematics and Statistics

SOUTHWEST UNIVERSiTy

Chongqing 400715, P. R. China

AND

DEPARTMENT OF SCIENCE

Sichuan University of Science And Engineering

Zigong 643000, P. R. ChinA

E-mail address: lilin420@gmail.com 